\title{
Irregular nonlinear operator equations: Tikhonov's regularization and iterative approximation
}

\author{
Vladimir Vasin
}

\begin{abstract}
A problem of iterative approximation is investigated for a nonlinear operator equation regularized by the Tikhonov method. The Levenberg-Marquardt method, its modified analogue, and the steepest descent method are used. For the first and second methods the regularizing properties of iterations are established and the error of approximate solution is given. For the third method it was proved that iterations are stabilized in a neighborhood of the required solution and satisfy the strong Fejér property.
\end{abstract}

Keywords. Levenberg-Marquardt method, steepest descent method, convergence rate, source condition, iterative regularization.

2010 Mathematics Subject Classification. 65J15, 65J20.

\section{Introduction}

The nonlinear operator equation is considered:

$$
A(u)=f
$$

with a Frechét differentiable operator $A$ acting on a pair of Hilbert spaces $U, F$. Continuity of operators $A^{-1}$ or $A^{\prime}(u)^{-1}$ is not assumed in a neighborhood of a solution. So, equation (1.1) defines an ill-posed (irregular) problem. Note that the operator equation is a sufficiently general form for the presentation of inverse problems, and such problems can be seen through all fields of natural science where methods of mathematical modeling are used.

Unboundedness of the operator $A^{\prime}(u)^{-1}$ excludes the opportunity of using iterative methods of the Newton, Gauss-Newton, or Levenberg-Marquardt type for approximating a solution of equation (1.1). So, in such a case, it is necessary either to pass to regularized analogues of the mentioned methods on the basis of the

The research was performed in the Ural Federal University and is supported by grant of the Russia Federation Government (Agreement no. 11.G34.31.0064), and also, it is partially supported by Ural Branch of RAS (project 12-P-15-2019) and Russian Foundation for Basic Research (project 12-0100106). 
iterative regularization principle or to apply these iterative processes not to (1.1), but to the regularized equation

$$
A^{\prime}(u)^{*}\left(A(u)-f_{\delta}\right)+\alpha\left(u-u^{*}\right)=0
$$

for some fixed and appropriately chosen parameter of regularization $\alpha$ and initial guess $u^{*}$; here, $\left\|f-f_{\delta}\right\| \leqslant \delta$.

It is necessary to note that in solving equation (1.1) by the Levenberg-Marquardt method (ML-M)

$$
u^{k+1}=u^{k}-\left[A^{\prime}\left(u^{k}\right)^{*} A^{\prime}\left(u^{k}\right)+\alpha I\right]^{-1} A^{\prime}\left(u^{k}\right)^{*}\left(A\left(u^{k}\right)-f_{\delta}\right)
$$

one must impose rather heavy structural conditions on the operator $A$ and on the choice of the regularizing parameter $[6,7,9,18]$.

In $[6,7]$ (see also [9]), it was suggested the following rule for choosing the parameter in the method (1.3), i.e., the parameter $\alpha=\alpha_{k}$ would be such that the following relation holds:

$$
\left\|f_{\delta}-A\left(u^{k}\right)-A^{\prime}\left(u^{k}\right)\left(u^{k+1}(\alpha)-u^{k}\right)\right\|=q\left\|f_{\delta}-A\left(u^{k}\right)\right\|,
$$

where $0<q<1$. Under this, existence of a unique solution $\alpha=\alpha_{k}$ for equation (1.3) is guaranteed if the following inequality holds:

$$
\left\|f_{\delta}-A\left(u^{k}\right)-A^{\prime}\left(u^{k}\right)\left(z-u^{k}\right)\right\|<q\left\|f_{\delta}-A\left(u^{k}\right)\right\|,
$$

where $z$ is a solution for equation (1.1) of the minimal norm.

The strong convergence of method (1.3) to a solution of equation (1.1) was proved under the condition that the operator $A$ satisfies the property

$$
\left\|A(u)-A(\tilde{u})-A^{\prime}(u)(u-\tilde{u})\right\| \leqslant C\|A(u)-A(\tilde{u})\|
$$

in some neighborhood $O_{r}\left(u^{0}\right)=\left\{u:\left\|u-u^{0}\right\| \leqslant r\right\}$ of a probe solution $u^{0}$, see $[7,9]$.

In the paper [18], two variants of ML-M were considered. The first one has the form

$$
u^{k+1}=u^{k}-\beta\left[A^{\prime}\left(u^{k}\right)^{*} A^{\prime}\left(u^{k}\right)+\alpha I\right]^{-1} A^{\prime}\left(u^{k}\right)^{*}\left(A\left(u^{k}\right)-f\right) \equiv T\left(u^{k}\right),
$$

i.e., in method (1.3), a positive parameter $\beta$ is introduced. In the second variant (as an analogy to the modified Newton method), the derivative in the inverse operator is calculated at a fixed point $u^{0}$, namely,

$$
u^{k+1}=u^{k}-\beta\left[A^{\prime}\left(u^{0}\right)^{*} A^{\prime}\left(u^{0}\right)+\alpha I\right]^{-1} A^{\prime}\left(u^{k}\right)^{*}\left(A\left(u^{k}\right)-f\right) \equiv T_{0}\left(u^{k}\right) .
$$

It was established that the operators $T, T^{0}$ are pseudo-contracting if the local condition

$$
\|A(u)-f\|^{2} \leqslant \varkappa\left\langle B^{-1}(u) S(u), u-z\right\rangle
$$


is satisfied, where

$$
S(u)=A^{\prime}(u)^{*}(A(u)-f), \quad B^{-1}(u)=\left(A^{\prime}(u)^{*} A^{\prime}(u)+\alpha I\right)^{-1},
$$

for method (1.5) and the condition

$$
\|A(u)-f\|^{2} \leqslant \varkappa\langle S(u), u-z\rangle
$$

for method (1.6) under $\beta<\beta_{0}(\varkappa)$ in some neighborhood $O_{\rho}(z)$ of a solution for equation (1.1). This property of the operators $T, T_{0}$ implies a weak convergence of iterations to a solution of this equation for exact input data.

Thus, to substantiate convergence of the MM-L to a solution of equation (1.1), rather heavy conditions (of the forms (1.4) or (1.7), or (1.8)) must be satisfied for the operator $A$.

By these reasons, the passage from equation (1.1) to the regularized equation (1.2) is fruitful since the operator

$$
S_{\alpha}(u)=A^{\prime}(u)^{*}\left(A(u)-f_{\delta}\right)+\alpha\left(u-u^{*}\right),
$$

which is the gradient of the Tikhonov functional

$$
\Phi(u)=\frac{1}{2}\left\|A(u)-f_{\delta}\right\|^{2}+\alpha\left\|u-u^{*}\right\|^{2},
$$

has (under some conditions) the better structural properties than $A$ and $A^{\prime *} A$. For example, if relations (1.12) and (1.13) hold, then the operator $S_{\alpha}$ have the property of strong monotonicity $[10,11]$, but otherwise for $A$ and $A^{\prime *} A$ this property does not hold.

It allows one to provide the strong convergence of the iterative processes for equation (1.2). Together with the Tikhonov regularization, this gives an opportunity to build a regularizing algorithm for the initial problem (1.1), in particular, for some classes of inverse problems in geophysics [18, 19].

In the author's work [14], the iterative ML-M process is investigated in the form

$$
u_{\alpha}^{k+1}=u_{\alpha}^{k}-\gamma\left[A^{\prime}\left(u_{\alpha}^{k}\right)^{*} A^{\prime}\left(u_{\alpha}^{k}\right)+\bar{\alpha} I\right]^{-1} S_{\alpha}\left(u_{\alpha}^{k}\right)
$$

and its modified variant in the form

$$
u_{\alpha}^{k+1}=u_{\alpha}^{k}-\gamma\left[A^{\prime}\left(u_{\alpha}^{0}\right)^{*} A^{\prime}\left(u_{\alpha}^{0}\right)+\bar{\alpha} I\right]^{-1} S_{\alpha}\left(u_{\alpha}^{k}\right)
$$

for the approximation of the solution $u_{\alpha}$ for the regularized equation (1.2). It was proved there that under the following conditions on the operator,

$$
\left\|A^{\prime}(u)\right\| \leqslant N_{1}, \quad\left\|A^{\prime}(u)-A^{\prime}(v)\right\| \leqslant N_{2}\|u-v\|,
$$


$\gamma \leqslant \gamma(\alpha, \bar{\alpha})$ and the sourcewise represented solution

$$
z-u^{*}=A^{\prime}(z)^{*} v
$$

for iterations $u_{\alpha}^{k}$ of processes (1.10), (1.11) strongly converge to the solution $u_{\alpha}$ of equation (1.2). Namely, the following theorems hold [14].

Theorem 1.1. Let conditions (1.12) and (1.13) be satisfied, and, moreover, let $\delta \leqslant \alpha /\left(12 N_{2}\right), \bar{\alpha} \geqslant 3 N_{1}^{2}, \alpha<\left(1-N_{2}\|v\|\right) N_{1}^{2}, r=\alpha /\left(12 N_{1} N_{2}\right)$. Then under $\gamma<\alpha /(2 \bar{\alpha})$ for any initial guess $u_{\alpha}^{0} \in S_{\alpha}\left(u_{\alpha}\right)$, the iterative process (1.10) converges to the regularized solution $u_{\alpha}$ and the strong Fejér property holds

$$
\left\|u_{\alpha}^{k+1}-u_{\alpha}\right\|^{2} \leqslant\left\|u_{\alpha}^{k}-u_{\alpha}\right\|^{2}-v\left\|u_{\alpha}^{k+1}-u_{\alpha}^{k}\right\|^{2}, \quad v=\alpha /(2 \bar{\alpha} \gamma)-1 .
$$

Theorem 1.2. Let conditions of Theorem 1.1 be satisfied. Then under $\gamma<2 \alpha /(3 \bar{\alpha})$ for any initial guess $u_{\alpha}^{0} \in S_{r}\left(u_{\alpha}\right)$, the iterative process (1.11) converges to the regularized solution $u_{\alpha}$ and the strong Fejér property holds under $v=2 \alpha /(\gamma 3 \bar{\alpha})-1$. Moreover, under special dependence $\alpha(\delta)$ beginning from some $k \geqslant k_{0}$, all iteration points $u_{\alpha}^{k}$ belong to some neighborhood $O_{r}(z)$ of a radius $r=c \delta^{1 / 2}$ of the solution $z$.

Properties of the iterations of processes (1.10) and (1.11) established in Theorems 1.1 and 1.2 are consequences of the strong Fejér property of the step operators in these methods. Note that (see $[15,16])$ the class of operators with such a property is closure with respect to operations of multiplication and convex summation. This allows one:

(1) to build new classes of hybrid iterative processes for solving the initial problem (1.1),

(2) to construct the step operator of the main process in the form of superposition of operators responsible for approximation of solutions of some sub-problems that compile the initial problem,

(3) in an economical way to take into account additional a priori constraints onto the required solution in the resolving iterative process.

In Section 2 of this work, we show that under $\gamma=1$ the iterations converge with the geometric rate and satisfaction of conditions (1.12) and (1.13) in a sufficiently small neighborhood of the regularized solution $u_{\alpha}$. Together with results from the book [4], it implies an one-step regularizing algorithm with the estimate of an approximate solution. This estimate is coordinated with one of the LevenbergMarquardt method obtained in [3] under more strong conditions onto the operator and sourcewise represented solution in the form

$$
z-u^{*}=f\left(A^{\prime}(z)^{*} A^{\prime}(z)\right) w
$$


for $f(t)=t^{\mu}, \mu=1$, with another choice of the regularizing parameter and the rule for stopping the iteration process. Note that the logarithmic function is also used in estimate of errors in in several works (see [3] and the bibliography there).

In Section 3, the steepest descent method is considered under the same conditions (1.12) and (1.13) for approximating the regularized solution. The regularized version of the steepest descent method is built; moreover, the strong convergence of iterations is proved and their stabilization is established in a neighborhood of a solution of the initial equation.

In conclusion of this section, let us give one result from the book [4] that touches the error estimate in the Tikhonov method, since we shall use it in further sections.

Lemma 1.3 ([4]). Let equation (1.2) be solvable and conditions (1.12), (1.13) be satisfied, where $\|v\|<1 / N_{2}$. Then for the solution $u_{\alpha}$ of equation (1.2) the following estimates hold:

$$
\begin{aligned}
\left\|A\left(u_{\alpha}\right)-f_{\delta}\right\| & \leqslant \delta+2 \alpha\|v\|, \\
\left\|u_{\alpha}-z\right\| & \leqslant \frac{\delta+\alpha\|v\|}{\sqrt{\alpha\left(1-N_{2}\|v\|\right)}} .
\end{aligned}
$$

\section{Convergence of iterations and error estimate}

2.1. Investigate now the iterative processes (1.10) for the approximation of a solution of the regularized equation (1.2) assuming only that conditions of type (1.12) and (1.13) are satisfied for the operator $A$.

Theorem 2.1. Let the operator $A$ be Frechet differentiable and estimates (1.12) hold for norms of derivatives in some neighborhood $O_{r}\left(u_{\alpha}\right)$. Let for a solution $z$ of equation (1.1) and a probe solution $u^{*}$ representation (1.13) be valid and the solution $u_{\alpha}$ of the regularized equation (1.2) exist. Then if the parameters $\alpha, \bar{\alpha}$, and $\delta$ and the initial approximations $u^{*}$ and $u_{\alpha}^{0}$ are such that

$$
\begin{gathered}
\bar{\alpha} \geq \alpha, \quad\left\|u_{\alpha}^{0}-u_{\alpha}\right\| \leqslant r, \\
\frac{1}{\bar{\alpha}}\left[(\bar{\alpha}-\alpha)+\frac{1}{2} N_{1} N_{2} r+N_{2}(\delta+2 \alpha\|v\|)\right]=q<1,
\end{gathered}
$$

then for method (1.10) under $\gamma=1$, the following estimate holds:

$$
\left\|u_{\alpha}^{k+1}-u_{\alpha}\right\| \leqslant q\left\|u^{k}-u_{\alpha}\right\| \leqslant q^{k+1} r,
$$

Proof. In the first part, let us use the scheme for the error estimation from the work [13] under some weaker assumptions. According to conditions of the theo- 
rem the following relations hold:

$$
\begin{gathered}
S_{\alpha}\left(u_{\alpha}\right)=A^{\prime}\left(u_{\alpha}\right)^{*}\left(A\left(u_{\alpha}\right)-f_{\delta}\right)+\alpha\left(u_{\alpha}-u^{*}\right)=0 \\
A\left(u_{\alpha}\right)=A\left(u_{\alpha}^{k}\right)+A^{\prime}\left(u_{\alpha}^{k}\right)\left(u_{\alpha}-u_{\alpha}^{k}\right)+\xi, \quad\|\xi\| \leqslant \frac{1}{2} N_{2}\left\|u_{\alpha}-u_{\alpha}^{k}\right\| .
\end{gathered}
$$

Taking into account relations (2.4), (2.5), the estimate

$$
\left\|A^{\prime}\left(u_{\alpha}^{k}\right)^{*} A^{\prime}\left(u_{\alpha}^{k}\right)+\bar{\alpha} I\right\|^{-1} \leqslant \frac{1}{\bar{\alpha}}
$$

and the theorem conditions, we have

$$
\begin{gathered}
\left\|u_{\alpha}^{k+1}-u_{\alpha}\right\|=\|\left(u_{\alpha}^{k}-u_{\alpha}\right)-\left[A^{\prime}\left(u_{\alpha}^{k}\right)^{*} A^{\prime}\left(u_{\alpha}^{k}\right)+\bar{\alpha} I\right]^{-1}\left[A^{\prime}\left(u_{\alpha}^{k}\right)^{*}\left(A\left(u_{\alpha}^{k}\right)-f_{\delta}\right)\right. \\
\left.+\alpha\left(u_{\alpha}^{k}-u^{*}\right)-A^{\prime}\left(u_{\alpha}\right)^{*}\left(A\left(u_{\alpha}\right)-f_{\delta}\right)-\alpha\left(u_{\alpha}-u^{*}\right)\right] \| \\
=\|\left(u_{\alpha}^{k}-u_{\alpha}\right)-\left[A^{\prime}\left(u_{\alpha}^{k}\right)^{*} A^{\prime}\left(u_{\alpha}^{k}\right)+\bar{\alpha} I\right]^{-1}\left[A^{\prime}\left(u_{\alpha}^{k}\right)^{*}\left(A\left(u_{\alpha}\right)-f_{\delta}\right)\right. \\
\quad+A^{\prime}\left(u_{\alpha}^{k}\right)^{*} A^{\prime}\left(u_{\alpha}^{k}\right)\left(-u_{\alpha}+u_{\alpha}^{k}\right)-A^{\prime}\left(u_{\alpha}^{k}\right)^{*} \xi+\alpha\left(u_{\alpha}^{k}-u^{*}\right) \\
\left.\quad-A^{\prime}\left(u_{\alpha}\right)^{*}\left(A\left(u_{\alpha}\right)-f_{\delta}\right)-\alpha\left(u_{\alpha}-u^{*}\right)\right] \| \\
=\|\left(u_{\alpha}^{k}-u_{\alpha}\right)-\left[A^{\prime}\left(u_{\alpha}^{k}\right)^{*} A^{\prime}\left(u_{\alpha}^{k}\right)+\bar{\alpha} I\right]^{-1} \\
\quad \times\left[A^{\prime}\left(u_{\alpha}^{k}\right)^{*} A^{\prime}\left(u_{\alpha}^{k}\right)+\bar{\alpha} I\right]\left(u_{\alpha}^{k}-u_{\alpha}\right) \\
\quad-(\bar{\alpha}-\alpha)\left(u_{\alpha}^{k}-u_{\alpha}\right)-A^{\prime}\left(u_{\alpha}^{k}\right)^{*} \xi \\
\left.\quad+\left(A^{\prime}\left(u_{\alpha}^{k}\right)^{*}-A^{\prime}\left(u_{\alpha}\right)^{*}\right)\left(A\left(u_{\alpha}\right)-f_{\delta}\right)\right] \| \\
\leqslant(1 / \bar{\alpha})\left[(\bar{\alpha}-\alpha)+\frac{1}{2} N_{1} N_{2}\left\|u_{\alpha}^{k}-u_{\alpha}\right\|\right. \\
\left.\quad+N_{2}\left\|A\left(u_{\alpha}\right)-f_{\delta}\right\|\right] \cdot\left\|u_{\alpha}^{k}-u_{\alpha}\right\| .
\end{gathered}
$$

Using relation (1.14) for the regularizing solution, we obtain at last

$$
\begin{aligned}
\left\|u_{\alpha}^{k+1}-u_{\alpha}\right\| & \leqslant \frac{1}{\bar{\alpha}}\left[(\bar{\alpha}-\alpha)+\frac{1}{2} N_{1} N_{2} r+N_{2}(\delta+2 \alpha\|v\|)\right]\left\|u_{\alpha}^{k}-u_{\alpha}\right\| \\
& =q\left\|u_{\alpha}^{k}-u_{\alpha}\right\|,
\end{aligned}
$$

i.e., estimate (2.3).

Corollary 2.2. Assume that $r=\alpha /\left(3 N_{1} N_{2}\right), \delta \leqslant \alpha /\left(6 N_{2}\right),\|v\| \leqslant 1 /\left(12 N_{2}\right)$ and $\left\|u_{\alpha}^{0}-u_{\alpha}\right\| \leqslant r, \bar{\alpha} \geq \alpha$. Then for method (1.10) under $\gamma=1$, all iterations $u_{\alpha}^{k}$ belong to the ball $S_{r}\left(u_{\alpha}\right)$ and the value $q=1-\alpha /(2 \bar{\alpha})<1$, i.e., the iterative process converges with the geometric rate. 
For the proof, it is sufficient to substitute the majorants for entering parameters into the above obtained estimate.

2.2. Now consider the modified Levenberg-Marquardt method (1.11).

Theorem 2.3. Let the conditions of Theorem 2.1 be satisfied. Then if the parameters $\alpha, \bar{\alpha}, \delta$, and the initial approximations $u^{*}, u_{\alpha}^{0}$, v are such that $\left\|u_{\alpha}^{0}-u_{\alpha}\right\| \leqslant r$, $\bar{\alpha} \geq \alpha$ and the relation

$$
\frac{1}{\bar{\alpha}}\left[(\bar{\alpha}-\alpha)+\frac{9}{2} N_{1} N_{2} r+N_{2}(\delta+2 \alpha\|v\|)\right]=q<1
$$

is valid, then for method (1.11) under $\gamma=1$, the following estimate is valid:

$$
\left\|u_{\alpha}^{k+1}-u_{\alpha}\right\| \leqslant \bar{q}\left\|u_{\alpha}^{k}-u_{\alpha}\right\| \leqslant \bar{q}^{k+1} r .
$$

Proof. By the conditions of the theorem there holds $\left\|u_{\alpha}^{0}-u_{\alpha}\right\| \leqslant r$. Assume that $\left\|u_{\alpha}^{k}-u_{\alpha}\right\| \leqslant r$. Modifying a little the estimation scheme from Theorem 2.1 and using relations (1.12), (1.13), (2.4), and (2.5), we obtain

$$
\begin{gathered}
\left\|u_{\alpha}^{k+1}-u_{\alpha}\right\|=\|\left(u_{\alpha}^{k}-u_{\alpha}\right)-\left[A^{\prime}\left(u_{\alpha}^{0}\right)^{*} A^{\prime}\left(u_{\alpha}^{0}\right)+\bar{\alpha} I\right]^{-1}\left[A^{\prime}\left(u_{\alpha}^{k}\right)^{*}\left(A\left(u_{\alpha}^{k}\right)-f_{\delta}\right)\right. \\
\left.+\alpha\left(u_{\alpha}^{k}-u^{*}\right)-A^{\prime}\left(u_{\alpha}\right)^{*}\left(A\left(u_{\alpha}\right)-f_{\delta}\right)-\alpha\left(u_{\alpha}-u^{*}\right)\right] \| \\
=\|\left(u_{\alpha}^{k}-u_{\alpha}\right)-\left[A^{\prime}\left(u_{\alpha}^{0}\right)^{*} A^{\prime}\left(u_{\alpha}^{0}\right)+\bar{\alpha} I\right]^{-1}\left[A^{\prime}\left(u_{\alpha}^{k}\right)^{*}\left(A\left(u_{\alpha}\right)-f_{\delta}\right)\right. \\
\quad-A^{\prime}\left(u_{\alpha}^{k}\right)^{*} A^{\prime}\left(u_{\alpha}^{k}\right)\left(u_{\alpha}-u_{\alpha}^{k}\right)-A^{\prime}\left(u_{\alpha}^{k}\right)^{*} \xi+\alpha\left(u_{\alpha}^{k}-u_{\alpha}\right) \\
\left.\quad-A^{\prime}\left(u_{\alpha}\right)^{*}\left(A\left(u_{\alpha}\right)-f_{\delta}\right)\right] \| \\
=\|\left(u_{\alpha}^{k}-u_{\alpha}\right)-\left[A^{\prime}\left(u_{\alpha}^{0}\right)^{*} A^{\prime}\left(u_{\alpha}^{0}\right)+\bar{\alpha} I\right]^{-1} \\
\quad \times\left[A^{\prime}\left(u_{\alpha}^{0}\right)^{*} A^{\prime}\left(u_{\alpha}^{0}\right)\left(u_{\alpha}^{k}-u_{\alpha}\right)+\bar{\alpha}\left(u_{\alpha}^{k}-u_{\alpha}\right)\right. \\
\quad+\left(A^{\prime}\left(u_{\alpha}^{k}\right)^{*} A^{\prime}\left(u_{\alpha}^{k}\right)-A^{\prime}\left(u_{\alpha}^{0}\right)^{*} A^{\prime}\left(u_{\alpha}^{0}\right)\right)\left(u_{\alpha}-u_{\alpha}^{k}\right) \\
\quad-(\bar{\alpha}-\alpha)\left(u_{\alpha}^{k}-u_{\alpha}\right) \\
\left.\quad+\left(A^{\prime}\left(u_{\alpha}^{k}\right)^{*}-A^{\prime}\left(u_{\alpha}\right)^{*}\right)\left(A\left(u_{\alpha}\right)-f_{\delta}\right)-A^{\prime}\left(u_{\alpha}^{k}\right)^{*} \xi\right] \| \\
\leqslant \frac{1}{\bar{\alpha}}\left[(\bar{\alpha}-\alpha)+2 N_{1} N_{2}\left\|u_{\alpha}^{k}-u_{\alpha}^{0}\right\|+N_{2}\left\|A\left(u_{\alpha}\right)-f_{\delta}\right\|\right. \\
\left.\quad+\frac{1}{2} N_{1} N_{2}\left\|u_{\alpha}^{k}-u_{\alpha}\right\|\right] \cdot\left\|u_{\alpha}^{k}-u_{\alpha}\right\| .
\end{gathered}
$$

Taking into account

$$
\left\|u_{\alpha}^{k}-u_{\alpha}^{0}\right\| \leqslant\left\|u_{\alpha}^{k}-u_{\alpha}\right\|+\left\|u_{\alpha}^{0}-u_{\alpha}\right\| \leqslant 2 r
$$


and

$$
\left\|A\left(u_{\alpha}\right)-f_{\delta}\right\| \leqslant \delta+2 \alpha\|v\|,
$$

we obtain the required estimate

$$
\left\|u_{\alpha}^{k+1}-u_{\alpha}\right\| \leqslant \bar{q}\left\|u_{\alpha}^{k}-u_{\alpha}\right\| .
$$

This completes the proof of the theorem.

Corollary 2.4. Let $r=\alpha /\left(12 N_{1} N_{2}\right), \delta \leqslant \alpha /\left(6 N_{2}\right),\|v\| \leqslant 1 /\left(12 N_{2}\right)$. Then for the modified Levenberg-Marquardt process (1.11) under $\gamma=1$, all iterations $u_{\alpha}^{k}$ belong to the ball $S_{r}\left(u_{\alpha}\right)$ and in estimate (2.6) the value $\bar{q}=1-\frac{7 \alpha}{24 \bar{\alpha}}<1$.

2.3. Gathering estimates (1.14), (1.15) for the regularized solution and estimates (2.3), (2.6) obtained for the iterative processes (1.10), (1.11), we come to the following state.

Theorem 2.5. Let the conditions of Theorem 2.1 and Corollary 2.2 (of Theorem 2.3 and Corollary 2.4) be satisfied and $\alpha(\delta)=c \delta$. Then by choice of the iteration number

$$
k(\delta)=\frac{\ln \left(\rho \delta^{1 / 2} / r\right)}{\ln (q)},
$$

where $q=1-\alpha /(2 \bar{\alpha})(q=1-7 \alpha /(24 \bar{\alpha})), \delta<(r / \rho)^{2}$, the following estimate is valid for methods (1.10) and (1.11) under $\gamma=1$ :

$$
\left\|u_{\alpha(\delta)}^{k(\delta)}-z\right\| \leqslant 2 \rho \delta^{1 / 2}
$$

where $\rho=(1+\|v\|) / \sqrt{c\left(1-N_{2}\|v\|\right)}$.

Proof. In the relation

$$
\left\|z-u_{\alpha}^{k}\right\| \leqslant\left\|z-u_{\alpha}\right\|+\left\|u_{\alpha}-u_{\alpha}^{k}\right\|
$$

for the first summand on the right-hand side of the inequality under (1.15) and $\alpha(\delta)=c \delta$, we have

$$
\left\|u_{\alpha(\delta)}-z\right\| \leqslant \frac{1+\|v\|}{\sqrt{c\left(1-N_{2}\|v\|\right)}} \cdot \delta^{1 / 2} .
$$

For the second summand by (2.3), (2.6), the following estimate holds:

$$
\left\|u_{\alpha(\delta)}^{k}-u_{\alpha(\delta)}\right\| \leqslant q^{k} \cdot r .
$$


Substituting (2.10) and (2.11) into (2.9), we can define the number of iterations under given $\delta$ from the condition

$$
\rho \delta^{1 / 2}=r q^{k} .
$$

Namely, we obtain (2.7) for the number of iterations and estimate (2.8) for the regularized family of the approximate solutions $\left\{u_{\alpha(\delta)}^{k(\delta)}\right\}$ in problem (1.1).

Remark 2.6. Estimates (2.8) and (2.10) are optimal by the order of $\delta$. It is true since under the same assumptions (1.13) in the linear case of the self-conjugate positive-define operator $A$, the modulus of continuity of the inverse operator $A^{-1}$ has the same order. Remind that namely the modulus of continuity of the inverse operator is used for estimating from below the error of the optimal method (see the states in [8, pages 138 and 178]).

Remark 2.7. In some applied problems, for example, in the structural inverse geophysical ones [18,19], estimates (1.12) for the derivatives of the operator $A$ do not hold for all elements $u \in U$, but are valid only on some convex closed set $Q$. To overcome this difficulty, it is sufficient to pass from (1.10) and (1.11) to the process of the form

$$
u_{\alpha}^{k+1}=P_{Q}\left(T\left(u_{\alpha}^{k}\right)\right),
$$

where $P_{Q}$ is the operator of metrical projection onto the set $Q$ and $T$ is the step operator for any of methods (1.10) or (1.11). If $z, u_{\alpha} \in Q$, then all states and estimates are also valid for (2.12).

\section{The steepest descent method (regularized version)}

3.1. To approximate the solution $u_{\alpha}$ of the regularized equation (1.2), consider the iterative process in the following form:

$$
u_{\alpha}^{k+1}=u_{\alpha}^{k}-\beta S_{\alpha}\left(u_{\alpha}^{k}\right),
$$

where the operator $S_{\alpha}$ is defined by formula (1.9). Linearizing equation (1.1) in the iteration point $u^{k}$,

$$
A(u) \simeq A\left(u^{k}\right)+A^{\prime}\left(u^{k}\right)\left(u-u^{k}\right),
$$

we come to the linear equation

$$
A^{\prime}\left(u^{k}\right) u=F,
$$

where $F=f-A\left(u^{k}\right)+A^{\prime}\left(u^{k}\right) u^{k}$. 
Since our interest is in approximation of the regularized equation, we shall find the value of parameter $\beta$ from the minimum of the regularized residual

$$
\min _{\beta}\left\{\left\|A^{\prime}\left(u^{k}\right)\left(u^{k}-\beta S_{\alpha}\left(u^{k}\right)\right)-F\right\|^{2}+\alpha\left\|u^{k}-\beta S_{\alpha}\left(u^{k}\right)-u^{*}\right\|^{2}\right\} .
$$

Using the necessary condition of the extremum, we obtain the value of the required parameter $\beta=\beta\left(u^{k}\right)$ :

$$
\beta\left(u^{k}\right)=\frac{\left\|S_{\alpha}\left(u_{\alpha}\right)\right\|^{2}}{\left\|A^{\prime}\left(u^{k}\right) S_{\alpha}\left(u^{k}\right)\right\|^{2}+\alpha\left\|S_{\alpha}\left(u^{k}\right)\right\|^{2}} .
$$

Note that in contrast to the traditional steepest descent method for the nonlinear operator equation (1.1) (see [12]), in the regularized version, the additional term with the coefficient $\alpha$ appears in the denominator for the parameter $\beta$.

Introduce the additional parameter $\gamma$ and consider the steepest descent method in the following form:

$$
u_{\alpha}^{k+1}=u_{\alpha}^{k}-\gamma \beta\left(u_{\alpha}^{k}\right) S\left(u_{\alpha}^{k}\right),
$$

where $\beta\left(u_{\alpha}^{k}\right)$ is defined by formula (3.2). It is easy to check out that conditions guaranteeing the geometric rate of convergence for process (3.3) are completely analogical to ones for the Landweber method obtained in the work [10]. Namely, if properties (1.12), (1.13) hold, then for $\delta<\alpha /\left(6 N_{2}\right),\|v\|<1 /\left(12 N_{2}\right)$ and the variable parameter taken as

$$
\gamma_{k}=\frac{d \alpha}{\beta\left(u_{\alpha}^{k}\right) M^{2}}, \quad 0<d<1,
$$

the following estimate is valid:

$$
\left\|u_{\alpha}^{k+1}-u_{\alpha}\right\| \leqslant q\left\|u_{\alpha}^{k}-u_{\alpha}\right\|,
$$

where $q=1-\alpha^{2}(-d+1) d / M^{2}<1, M=N_{1}^{2}+(4 / 3) \alpha$.

3.2. Now consider another opportunity for obtaining the strong convergence of process (3.3) that is based on the property of pseudo-contractibility of the step operator.

Theorem 3.1. Let the conditions (1.12), (1.13) be fulfilled and let $\delta \leqslant \alpha /\left(6 N_{2}\right)$, $\|v\| \leqslant 1 /\left(12 N_{2}\right), r=\alpha /\left(12 N_{1} N_{2}\right)$. Then for any initial guess $u_{\alpha}^{0} \in O_{r}\left(u_{\alpha}\right)$ under $\gamma<\alpha^{2} / M^{2}$, process (3.3) strongly converges to the regularized solution $u_{\alpha}$ and the strong Fejér condition holds for the iteration

$$
\left\|u_{\alpha}^{k+1}-u_{\alpha}\right\|^{2} \leqslant\left\|u_{\alpha}^{k}-u_{\alpha}\right\|^{2}-v\left\|u_{\alpha}^{k}-u_{\alpha}^{k+1}\right\|^{2} .
$$


Proof. Since $S_{\alpha}\left(u_{\alpha}\right)=0$ and estimates (1.12), (1.13) hold, we have under the conditions on the parameters $\delta,\|v\|, r$

$$
\begin{aligned}
\left\|S_{\alpha}(u)\right\|= & \left\|S_{\alpha}(u)-S_{\alpha}\left(u_{\alpha}\right)\right\| \\
= & \| A^{\prime}(u)^{*}\left(A(u)-f_{\delta}\right)+\alpha\left(u-u^{*}\right) \\
& \quad-A^{\prime}\left(u_{\alpha}\right)^{*}\left(A\left(u_{\alpha}\right)-f_{\delta}\right)+\alpha\left(u_{\alpha}-u^{*}\right) \| \\
\leqslant & \| A^{\prime}(u)^{*}\left(A(u)-A\left(u_{\alpha}\right) \|\right. \\
& \quad+\left\|\left(A^{\prime}(u)^{*}-A^{\prime}\left(u_{\alpha}\right)^{*}\right)\left(A\left(u_{\alpha}\right)-f_{\delta}\right)\right\|+\alpha\left\|u-u_{\alpha}\right\| \\
\leqslant & \left(N_{1}^{2}+(4 / 3) \alpha\right)\left\|u-u_{\alpha}\right\| .
\end{aligned}
$$

Under the conditions of this theorem on $u \in O_{r}\left(u_{\alpha}\right)=\left\{u:\left\|u-u_{\alpha}\right\| \leqslant r\right\}$, the following relation holds (see [10]):

$$
\left\langle S_{\alpha}(u), u-u_{\alpha}\right\rangle \geqslant \frac{\alpha}{2}\left\|u-u_{\alpha}\right\|^{2} .
$$

Joining (3.5) with (3.6), we come to the estimate

$$
\left\|S_{\alpha}(u)\right\|^{2} \leqslant \frac{2 M^{2}}{\alpha}\left\langle S_{\alpha}(u), u-u_{\alpha}\right\rangle
$$

where $M=N_{1}^{2}+(4 / 3) \alpha$.

The pseudo-contractibility (i.e., the strong Fejér property) for the step operator $T$ in process (3.3) means satisfaction of the inequality

$$
\left\|T(u)-u_{\alpha}\right\|^{2}-\left\|u-u_{\alpha}\right\|^{2}+v\|T(u)-u\|^{2} \leqslant 0
$$

for some $v>0$; and this with taking into account

$$
\beta(u)=\frac{\left\|S_{\alpha}(u)\right\|^{2}}{\left\|A^{\prime}(u) S_{\alpha}(u)\right\|^{2}+\alpha\left\|S_{\alpha}(u)\right\|^{2}} \geqslant \frac{\left\|S_{\alpha}(u)\right\|^{2}}{\left(\left\|A^{\prime}(u)\right\|^{2}+\alpha\right)\left\|S_{\alpha}(u)\right\|^{2}} \geq \frac{1}{N_{1}^{2}+\alpha}
$$

is equivalent to

$$
-\frac{2}{\gamma(1+v)}\left\langle u-u_{\alpha}, S_{\alpha}(u)\right\rangle+\frac{\left\|S_{\alpha}(u)\right\|^{4}}{\left\|A^{\prime}(u) S_{\alpha}(u)\right\|^{2}+\alpha\left\|S_{\alpha}(u)\right\|^{2}} \leqslant 0 .
$$

In turn, inequality (3.9) will be satisfied if

$$
\left\|S_{\alpha}(u)\right\|^{2} \leqslant \frac{2 \alpha}{\gamma(1+v)}\left\langle u-u_{\alpha}, S_{\alpha}(u)\right\rangle .
$$

Since estimate (3.7) holds, the pseudo-contractibility of the step operator in process (3.3) is guaranteed if $\gamma<\alpha^{2} / M^{2}$, and under this, for the given $\gamma$, we have

$$
v=-1+\frac{\alpha^{2}}{\gamma M^{2}} .
$$


Substituting $u=u_{\alpha}^{k}$ into (3.8), we obtain

$$
\left\|u_{\alpha}^{k+1}-u_{\alpha}\right\|^{2} \leqslant\left\|u_{\alpha}^{k}-u_{\alpha}\right\|^{2}-v\left\|u_{\alpha}^{k+1}-u_{\alpha}^{k}\right\|^{2},
$$

from which it follows

$$
\left\|u_{\alpha}^{k}\right\| \leqslant c, \quad \lim _{k \rightarrow \infty}\left\|u_{\alpha}^{k}-u_{\alpha}\right\|=d, \quad\left\|u_{\alpha}^{k+1}-u_{\alpha}^{k}\right\|=\gamma \beta\left(u_{\alpha}^{k}\right) S_{\alpha}\left(u_{\alpha}^{k}\right) \rightarrow 0,
$$

which together with (3.6) implies the convergence

$$
\lim _{k \rightarrow \infty}\left\|u_{\alpha}^{k}-u_{\alpha}\right\|=0 .
$$

3.3. In some important inverse problems (for example, in the inverse problems of gravimetry and magnetometry $[18,19])$ conditions (1.12) are not satisfied for all $u, v \in D(A) \subseteq U$. But it is possible to guarantee their satisfaction for elements from some convex set $Q$. In this case, one can slightly modify method (3.3) and obtain convergence for iterations.

Theorem 3.2. Let the conditions of Theorem 3.1 be satisfied with the difference that equations (1.12) hold only for $u, v \in Q$, where $Q$ is a convex, closed, and compact (optionally) subset of a Hilbert space $U$ containing $z, u_{\alpha}$. Then for the iterative process

$$
u_{\alpha}^{k+1}=P_{Q}\left[u_{\alpha}^{k}-\gamma \beta\left(u_{\alpha}^{k}\right) S_{\alpha}\left(u_{\alpha}^{k}\right)\right] \equiv P_{Q} T\left(u_{\alpha}^{k}\right),
$$

where $P_{Q}$ is the metric projection onto the set $Q$, the conclusion of Theorem 3.1 holds.

Proof. Since under the above conditions the step operator $T$ in process (3.3) is pseudo-contracting (see (3.8)) and, also, the projecting operator $[15,16]$ has the same property, the sequential application of this property to the operator $P_{Q} T$, we have

$$
\begin{aligned}
\left\|u_{\alpha}^{k+1}-u_{\alpha}\right\| & =\left\|P_{Q}\left(T\left(u_{\alpha}^{k}\right)\right)-u_{\alpha}\right\|^{2} \\
& \leqslant\left\|T\left(u_{\alpha}^{k}\right)-u_{\alpha}\right\|^{2}-\left\|P_{Q}\left(T\left(u_{\alpha}^{k}\right)\right)-T\left(u_{\alpha}^{k}\right)\right\|^{2} \\
& \leqslant\left\|u_{\alpha}^{k}-u_{\alpha}\right\|^{2}-v\left\|T\left(u_{\alpha}^{k}\right)-u_{\alpha}^{k}\right\|^{2}-\left\|P_{Q}\left(T\left(u_{\alpha}^{k}\right)\right)-T\left(u_{\alpha}^{k}\right)\right\|^{2},
\end{aligned}
$$

from which it follows

$$
\lim _{k \rightarrow \infty}\left\|u_{\alpha}^{k}-u_{\alpha}\right\|=d, \quad\left\|u_{\alpha}^{k}\right\| \leqslant \text { cerest }
$$

and

$$
\left\|T\left(u_{\alpha}^{k}\right)-u_{\alpha}^{k}\right\|=\gamma \beta\left(u_{\alpha}^{k}\right) S_{\alpha}\left(u_{\alpha}^{k}\right) \rightarrow 0, \quad k \rightarrow \infty .
$$

Taking into account that $\beta(u) \geqslant 1 /\left(N_{1}^{2}+\alpha\right)$, it follows from (3.11)

$$
S\left(u_{\alpha}^{k}\right) \rightarrow 0, \quad k \rightarrow \infty .
$$


Joining (3.6), (3.10), and (3.12), we obtain the convergence

$$
\lim _{k \rightarrow \infty}\left\|u_{\alpha}^{k}-u_{\alpha}\right\|=0
$$

together with the strong Fejér property of iterations (see relation (3.4)).

As it is known, to obtain convergence of the regularized solutions to $u^{*}$-normal exact solution $z$, it is sufficient to choose the regularizing parameter as follows: $\alpha(\delta)=c \delta^{\eta}, 0<\eta<2$. For definiteness, take $\alpha=c \delta$. Then on the basis of (1.15)

$$
\left\|u_{\alpha(\delta)}-z\right\| \leqslant x \delta^{1 / 2},
$$

where $\varkappa=(1+\|v\|) \sqrt{c\left(1-N_{2}\|v\|\right)}$. Since according to Theorem 3.1 and under the satisfaction of conditions (3.4) we have strong convergence of $u_{\alpha}^{k}$ to $u_{\alpha}$, all iterative points $u_{\alpha}^{k}$ (beginning from some $k \geqslant k_{0}$ ) enter the ball $O_{r}(z)$, where $r=\tau \varkappa \delta^{1 / 2}, \tau>1$. In this case, it is not necessary to formulate the rule for stopping the iterations.

Thus, the iterations generated by process (3.3) converge to $u_{\alpha(\delta)}$, which is some point from the neighborhood $O_{r}(z)$ of the exact solution $z$ with the radius $r=r(\delta)$, which decreases to zero together with $\delta \rightarrow 0$.

Remark 3.3. For the convergence of iterations (3.3), it is necessary to guarantee for the initial guess $u_{\alpha}^{0}$ to be in the ball $O_{r}\left(u_{\alpha}\right)$ of the radius $r=\alpha /\left(12 N_{1} N_{2}\right)$. Since $u_{\alpha}$ is unknown in computations, one can use estimate (3.6) to find an appropriate $u_{\alpha}^{0}$. Then, substituting $u=u_{\alpha}^{0}$ into (3.6), we obtain

$$
\frac{\alpha}{2}\left\|u_{\alpha}^{0}-u_{\alpha}\right\|^{2} \leqslant\left\langle S_{\alpha}\left(u_{\alpha}^{0}\right), u_{\alpha}^{0}-u_{\alpha}\right\rangle .
$$

Then $u_{\alpha}^{0}$ chosen by the condition

$$
\frac{2}{\alpha}\left\|S_{\alpha}\left(u_{\alpha}^{0}\right)\right\| \leqslant r
$$

can be a preliminary initial guess for solving the problem in spite of the fact that it does not guarantee the inclusion $u_{\alpha}^{0} \in O_{r}\left(u_{\alpha}\right)$.

Remark 3.4. Some particular implementations of method (1.10) $(\bar{\alpha}=\alpha, \alpha=0)$ were successfully applied to solving the inverse problems of geophysics $[18,19]$ and sounding the atmosphere in reconstructing the altitude profiles of temperature, methane, and the carbon dioxide by spectra of the high resolution (see [5, 17]). Note that in large scale (multi-variant) computations that are character in the inverse problems of the atmosphere sounding, the modified process (1.11) could be more effective. It is true since this process has the same rate of convergence as (1.10), but is more economic in computations, because it does not demand recalculations of the inverse operator (the matrix) on each iteration. 
Conclusion. The geometric rate of convergence to the regularized solution was established for the Levenberg-Marquardt method and its modified analogue in the case when the derivative of the problem operator satisfies condition (1.12) and the sourcewise representation of solution (1.13) holds.

It was proved that the iterations generate the regularizing algorithm for the initial problem with the error estimate that is optimal by the order. Under the same assumptions, it was proved for the regularized steepest descent method that the iterations converge to the regularized solution and stabilize in a neighborhood of the required solution with the radius proportional to the error of the right-hand side of the equation.

Thus, the suggested approach allows one to construct the strong approximation of the solution for the initial equation under comparatively non-burdensome conditions to the operator of problem (1.1). The approach is based on the preliminary regularization of the nonlinear operator equation and the iterative processes of the Levenberg-Marquardt and steepest descent types applied to the regularized equation.

\section{Bibliography}

[1] A. B. Bakushinsky, Methods of solving monotone variational inequalities based on principle of iterative regularization (in Russian), Zh. Vychisl. Mat. Mat. Fiz. 17 (1977), 1350-1362.

[2] A. B. Bakushinsky and A. V. Goncharsky, Ill-Posed Problems. Theory and Applications, Kluwer, Dordrecht, 1994.

[3] C. Böckmann, A. Kammanee and A. Braunb, Logarithmic convergence rate of Levenberg-Marquardt method with application to an inverse potential problem, J. Inverse Ill-Posed Probl. 19 (2011), 345-367.

[4] H. W. Engl, M. Hanke and A. Neubauer, Regularization of Inverse Problems, Kluwer, Dordrecht, 2000.

[5] K. G. Gribanov, V. I. Zakharov and V. V. Vasin, Iterative regularization in the problem finding concentration $\mathrm{CO}_{2}$ in atmosphere from data of satellite sounding, in: Abstracts of International Conference "Algorithmic Analysis of Unstable Problems" (Ekaterinburg 2008), IMM UrO RAN, Ekaterinburg (2008), 119-120.

[6] M. Hanke, A regularization Levenberg-Marquardt scheme, with applications to inverse groundwater filtration problems, Inverse Problems 13 (1997), 79-95.

[7] M. Hanke, The regularizing Levenberg-Marquardt scheme is of optimal order, J. Integral Equations Appl. 22 (2010), 259-283.

[8] V. K. Ivanov, V. V. Vasin and V.P. Tanana, Theory of Linear Ill-Posed Problems and Its Applications, VSP, Utrecht, 2002. 
[9] B. Kaltenbacher, A. Neubauer and O. Scherzer, Iterative Regularization Methods for Nonlinear Ill-Posed Problems, Walter de Gruyter, Berlin, 2008.

[10] M. Y. Kokurin, On convexity of the Tikhonov functional and iteratively regularized methods of solving irregular nonlinear operator equations (in Russian), Zh. Vychisl. Mat. Mat. Fiz. 50 (2010), 651-664.

[11] M. Y. Kokurin, On organization of global search under implementation of Tikhonov scheme (in Russian), Izv. Vuzov 12 (2010), 20-31.

[12] A. Neubauer and O. Scherzer, A convergence rate result for a steepest descent method and a minimal error method for the solution of nonlinear ill-posed problems, J. Anal. Appl. 14 (1995), 369-377.

[13] A. B. Smirnova and V. V. Vasin, Iterative approximation of solutions of nonlinear unstable problems in a Hilbert space, Russian J. Numer. Anal. Math. Modelling 8 (1993), no. 2, 127-144

[14] V. V. Vasin, The Levenberg-Marquardt method for approximation of solutions of irregular operator equations, Autom. Remote Control 73 (2012), no. 3, 440-449.

[15] V. V. Vasin and A. L. Ageev, Ill-Posed Problems with A Priori Information, VSP, Utrecht, 1995.

[16] V. V. Vasin and I. I. Eremin, Operators and Iterative Processes of Fejèr Type. Theory and Applications, Walter de Gruyter, Berlin, 2009.

[17] V. V. Vasin, K. G. Gribanov and V. I. Zakharov, Inverse problem of thermal sounding atmosphere (in Russian), Sib. Elektron. Mat. Izv. 5 (2008), 518-523.

[18] V. V. Vasin and G. Y. Perestoronina, The Levenberg-Marquardt method and its modified variant for solving nonlinear equations with application to the inverse gravimetry problem (in Russian), Trudy Inst. Mat. Mech. UrO RAN 17 (2011), 53-61.

[19] V. V. Vasin and G. G. Skorik, Iterative processes of gradient type with applications to gravimetry and magnetometry inverse problems, J. Inverse Ill-Posed Probl. 18 (2010), 855-876.

Received May 10, 2012.

\section{Author information}

Vladimir Vasin, Ural Federal University, Lenin Avenue, 51, Ekaterinburg 620000, Russia; and Institute of Mathematics and Mechanics UB RAS, S. Kovalevskay Street, 16, Ekaterinburg 620990, Russia.

E-mail: vasin@imm.uran.ru 
Copyright of Journal of Inverse \& Ill-Posed Problems is the property of De Gruyter and its content may not be copied or emailed to multiple sites or posted to a listserv without the copyright holder's express written permission. However, users may print, download, or email articles for individual use. 